\title{
Future Hydroponic Systems using IOT for sustainable agriculture
}

\author{
Sapna Jain $^{1}$, Prof M Afshar Alam², Prof M.U. Bokhari ${ }^{3}$ \\ \{drsapnajain@jamiahamdard.ac.in ${ }^{1}$,alam@jamiahamdard.ac.in ${ }^{2}, \underline{\text { mubokhari.cs@amu.ac.in }{ }^{3}}$ \} \\ Jamia Hamdard, New Delhi ${ }^{1,2}$, Aligarh Muslim University ${ }^{3}$
}

\begin{abstract}
Agriculture contributes to major part of economy of any country. The sustainable development of country needs equal development of infrastructure. We need an advance technology that can help to improve the quality and production of our crops. Hydroponics is a technique of crop soilless cultivation which is used worldwide. The paper discusses the use of latest IOT technology used with efficient hydroponic systems which help to save environment. This paper discusses how IoT provides a sustainable approach in agriculture sector where various technological devices use sensors and other tools for all the process of production of crops.
\end{abstract}

Keywords: smart agriculture, farming, Internet of Things, sustainable development, hydroponic systems

\section{Introduction}

Hydroponic systems are future sustainable agriculture systems. This method provides all the required nutrients. The system uses a timer controlled mechanism which is efficient in handling the pump and continuously check it . There are many types of hydroponic systems such as wick system , Flood and drain system, Aeroponic system . Hydroponic system can be easily built in indoors. The plants which use these systems yields much better results system[1]. These systems are based on a mechanism in which their roots can use oxygen efficiently by using less water which provides a sustainable method for production and harvesting of crops.

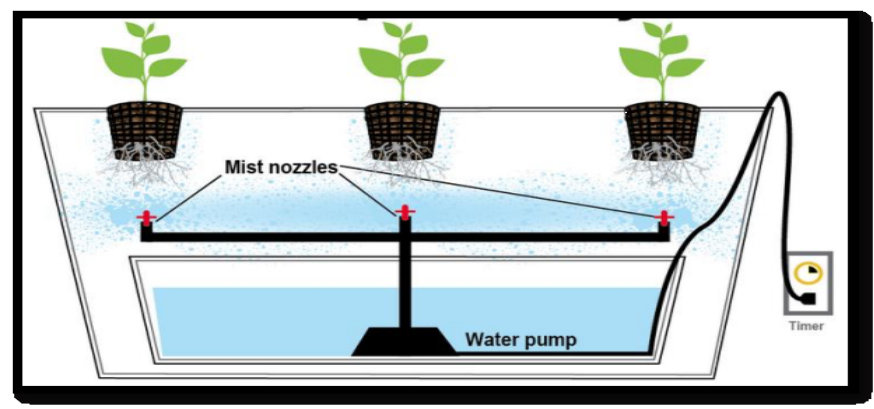

Figure 1. Hydroponic system

Source: Patil Anil, Sabale Snehal, Hydroponics farming using IOT, International journal of basic and applied research, May 2019 Volume 9 Number 5, www.pragatipublication.com ISSN 2249-3352 (P) 2278-0505 (E)

The purpose is to swing the roots in a way so they can get they can get the maximum amount of oxygen for the plant. Sublime amounts of oxygen receive roots from which plants take nutrition for growing fast which is method of hydroponic system to work[2].Generally very small plants are growing in soilless structure. The environment in which the plants can grow 
needs the use of small containers which is closed which flattens and cover the root of plants. Small holes are made around the head of the container in which the roots can easily lay down swinging growing in the chamber. The essential nutrients are sprayed and water is sprayed is prayed at regular intervals. The roots are always kept wet which helps in easy absorption of nutrients. Mounting chamber roots are kept in air tight compact environment. The plants gets the required oxygen and uses only required amount of water .The roots are kept moist. A water droplet is a component which sprinkle the required nutrients in proper and equal amount. The surrounding surface uses the oxygen with small streams of water using a sprinkler. The plant is growing rapidly as well as interpreted in the canopy[3,4].

\section{Background}

The hanging gardens of Babylon shows the use of hydroponic systems in ancient times. The Aztec floating gardens of Mexico and Old Chinese Cultures also show the use of hydroponic systems . William Gerricke in early 90's insisted the use hydroponic systems in the University of California at Berkeley to improve production These systems were soilless. Géricke became an inspiration for people who were doing tomato cultivation by growing tomatoes which is 25 feet high .This method used a small space in buy providing mineral nutrients without soil. Hydroponics had initial success on a place known as Wake Island. Hydroponics was there in 1930 for the passengers to grow vegetables due to its cost effective way of growing the fresh vegetables by using less oxygen and less water. These systems are had common errors is when growing and prevents it from underwater. Due to population explosion in recent years the land available for cultivation was scarce. To handle this situation NASA started experimentation and research in which they found that the hydroponic systems were able have to find suitable soil to grow crops. Plants also need supply of oxygen and environment to grow in $\mathrm{CO} 2$ free atmosphere.[4]

The research work done by Saeed $[5,6]$ states that hydroponic system uses the roots of the plant which is submerged in the nutrient and oxygen properly. A sensor is used to observe how much the nutrient content is used by plants. The research findings states the use of control sensor and microcontroller. The devices used helps to measure the growth with the help of sensors. The research discussed that the $\mathrm{pH}$ level changes shall starts when changes are done to show the effect of $\mathrm{pH}$ adjuster for using less water. The results showed that by adjusting the $\mathrm{pH}$ value in the water solution the appropriate nutrients was provided to the plant. This technique used the sensor to check the nutrient level .It helps to transfer the contents which helps to grow the plant. The system implementation results showed that the system is capable 0.58 decrease in use of oxygen and other nutrients. Lenord. [8] discussed a system which uses nutrient solution by using optimizing algorithm and parameters which helped in improving the production. The use of this method uses a some parameters and calculating function. Considerate amount of light helps the use of proper water and minerals used for growth of the plant. Fuzzy interference technique system was used by the expert researchers from Murugappa Chettiar Research Center in India. They made assystem using hydroponic technology which helps to evaluate the performance of the system. The implementation algorithm showed the less use of resources than earlier systems.

Rongsheng [9] suggested production of lettuce crop with the use red light. The use of different method helps to increase the growth. The results showed that the red light has increased with increasing intensity. The lysates were grown under separate light treatments for 35 days. The 
experimentation showed improvement of both the growth and quality of lettuce .The use of red light helped to increase the production. This study proved that red light was beneficial for growth and the quality of lettuce by using hydroponic method.

Saeed [10] showed that hydroponics systems uses sensor based programming which helps to improve production back process. It used to increase the crop production of spinach leafy vegetable with the use of water hydroponic plant. The use of this system shall help to understand the impact of different factors required to grow plant. The technique helped the methods to use organic farming in which the system uses possibilities of finding methods which help satisfy the requirements. The results shows the need the use sustainable technologies which helps the farmers to produce more output by putting less investment.

IOT uses sensors in agriculture which helps in decision support of smart farming creating a model in order to perform necessary tasks required for farmers was proposed with a intention to be applied to agriculture [11]. Agriculture IOT semantic framework is used on real time systems which supports reasoning over various heterogeneous sensor data streams. These systems can be used for smart farming which can combine multiple cross-domain data streams and semantic processing pipeline. The systems using large-scale data analytics online can add new features encouraging seam- less interoperability which can be used on open datasets and resources available on internet [12]. The specialized farm management modules offer characteristics which use generic software s which can be used easily by farmers.

[13]. The research work on autonomous gardening robotic vehicle helps to identify and classify plant species using feature extraction algorithms. These robotic systems uses

inputs such as temperature at which plant grows, humidity required, heat level required, the soil type and wind blowing direction appropriate for plants. The required data is collected from on-board sensors which use cloud storage platform and help in providing gardening

maintenance platform. All these systems related to historical work done,work required for maintenance and for future predictions is done more effectively and efficiently. The use of eresources includes use of a website and an android application which provide a platform for providing the monitoring of farms in remote areas[14]. The hydroponics farming using IOT based framework can effectively handle actual farm testbed performance applying for proposed frameworks. The use IOT and machine learning combined with data analytics can successfully help to monitor and govern the factors affecting the growth of plants [15].

\section{Internet of Things based Hydroponic Agriculture}

The sustainable agriculture using IOT based hydroponic systems have contributed in increasing the production of the following crops. The crops production of Lettuce, Fenugreek, spinach have increased by using the internet of things based Hydroponic systems.Lettuce cultivation is done in Astercase. This plant is used commonly for salad or as vegetable which are used as raw or cooked. In Europe and North America it is used as medicine. The plant is used for religious and medicinal purpose. The lettuce consumption of has constitute about 24.9 million tonnes which forms a major agriculture production. Fenugreek cultivation is done in South Asia .It is an annual plant with leaves[16].Make a crust of leaves consisting of three small oblates. It is used as seeds or a common ingredients in dishes from South Asia. This plant is cultivated in Iraq and ancient history. Fenugreek is believed to have been brought to close cultivation. Johri and Hoff recovered this leaf and its seeds $\mathrm{n} 4000 \mathrm{BC}$ which is bronze age. Spinach cultivation 
is done in Central and Western Asia .It is an edible flowering plant in the in which its leaves are eaten as a vegetable. The plant grows up to $30 \mathrm{~cm}(12 \mathrm{in})$ long. It is cultivated in winter regions and has different sizes. The sizes vary from $3-10 \mathrm{~mm}$ and is yellowish green in color. The plant has flowers with small leaves.

\subsection{IOT based Hydroponic System}

The System components diagram of the IoT based Hydroponic system is shown in the Figure 2.are [17]-

1. Power Supply.

2. Water Temperature and Level Sensor.

3. Microcontroller.

4. Light Sensor.

5. Relays to control lights, pumps.

6. WIFI Module.

7. Turbidity Sensor.

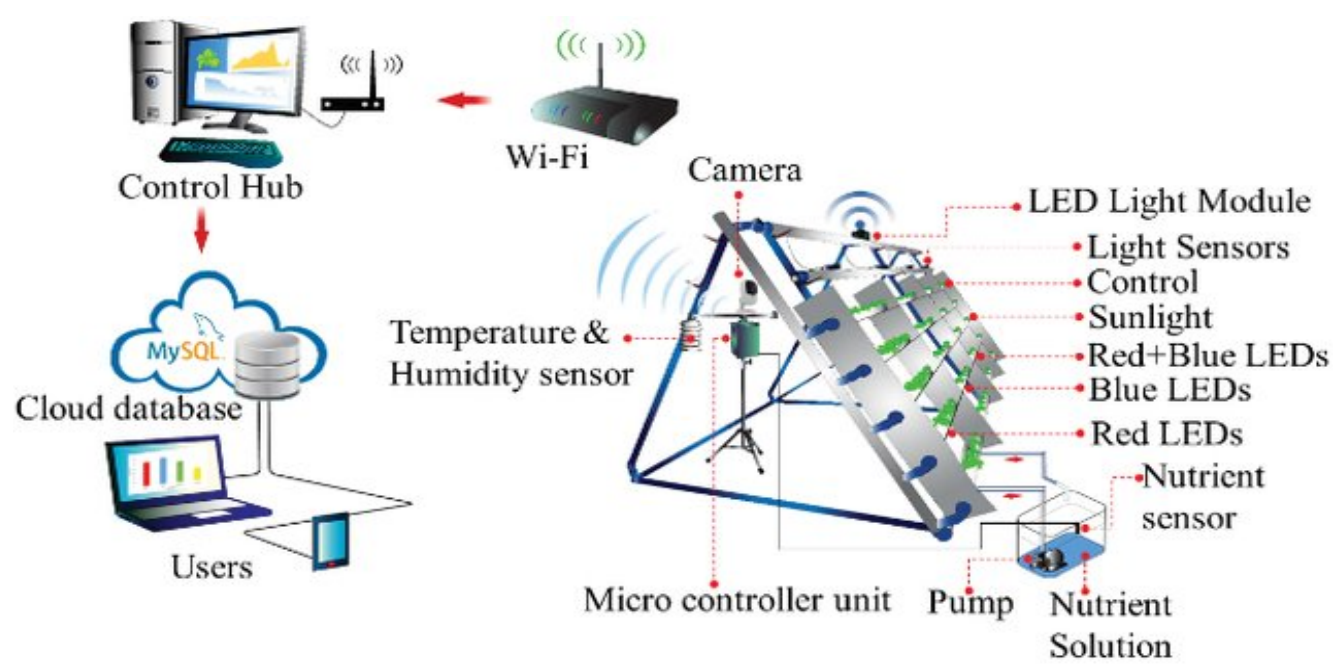

Figure 2. System IOT Components of Hyproponic System Source :T. Namgyel, S. Siyang, C. Khunarak, T. Pobkrut, J. Norbu, T. Chaiyasit, T. Kerdcharoen ,IoT based hydroponic system with supplementary LED light for smart home farming of lettuce, 2018 15th International Conference on Electrical Engineering/Electronics, Computer, Telecommunications and Information Technology 


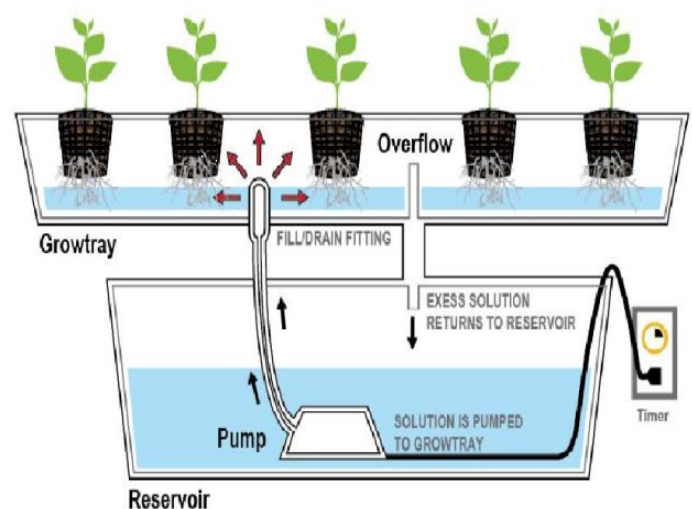

EBB \& FLOW CYCLE (PUMP ON)

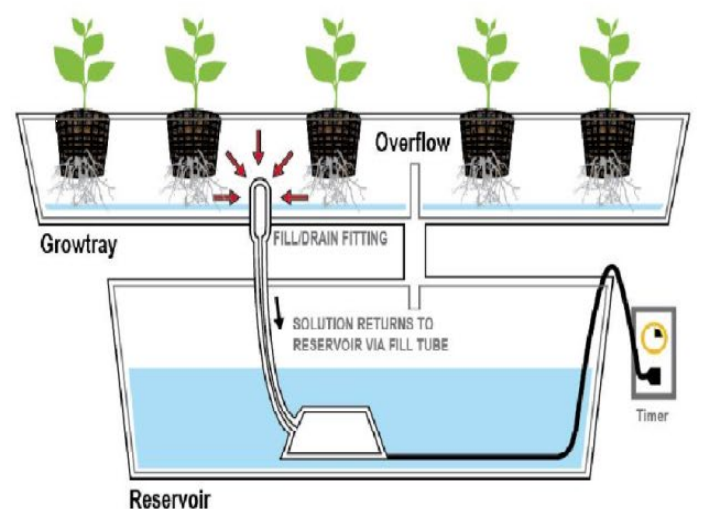

EBB \& FLOW CYCLE (PUMP OFF)

Figure 3. Work Flow of IOT based Hydroponic System

Source: Source : Chavan Akshay A, Pawar Abhijeet S, Wagh Pratik V, Prof. Lalita Wani , IOT Based Hydroponic System, International Journal of Innovative Research in Computer and Communication Engineering, Vol. 5, Issue 4, April 2017 , DOI: 10.15680/IJIRCCE.2017. 0504248

The work flow process in shown in Figure3.The nutrient is transported to the crop roots in the substrate and then filled into the tank. The pump turns on automatically and with the aid of a timer, the shutdown function is turned on. When there is a timer on the pump it starts transporting nutrients to the roots with the help of water. When it closes the solution goes into the tank by the drain outlet. The process is repeated after all solutions have gone into the tank. Flooding is done a few times a day which depends on the culture and substrate type. The loss is hidden in the lack of energy and breakdown of the pump.

\subsection{Advantages}

This system is based on usage of minimized resources. The devices required can be easily taken individually or by organisation. The farmer can easily access the different components by using less inputs. The farmer can be self-sustainable by using these components. The systems uses hardware like sensors and other technology devices. The requirements can be easily download and be used effectively. The components uses s soilless technology and consider the environment factors. These systems can be purchased by the farmer or any person easily a reasonable cost. A well-regulated hydroponic system can be very time-consuming to install and needs technical support. The systems needs to regularly check and monitor the use of consumption of nutrient levels. The use of IoT technology helps to in reducing the load of management significantly. These systems helps the farmers to become self-sustainable and contribute towards in achieving the sustainable development goals.

\section{Conclusion}

This paper explores the future hydroponically grown plants are exposed to use of oxygen and water. The required nutrients are given to the plants with required amount of water. The 
Hydroponic cultivation is done for many other crops and is used widely in developed and underdeveloped nations. Water consumption is also less which has made this type of farming popular. The use of these systems is the are required by various countries of the world .It has already been have proved that this technology is completely practical and has many advantages over traditional methods of crop production. These systems can help small scale farmers to move large systems. The IOT enabled systems uses stream of less water and stores the dissolved nutrients required for plant growth. These systems can provide soilless farming a popular and help to achieve the sustainable development goal 2 which is No Hunger End hunger and achieve food security.

\section{References}

[1] Mamta D. Sardare 1, Ms. Shraddha V. Admane ,"A review on plant without soil - hydroponics", International Journal research of engineering \& technology ,2013, Volume 2, Issue: 3

[2]Sahar Khan, Shreya Tembe, Rujuta Acharekar, "IoT based Automated Hydroponics System", International Journal of Scientific \& Engineering Research, 2018,Volume 2.

[3]S.Keerthana , K. Devika , S.Sathiyadevi, M. Priyanka," Automating and Analysing Greenhouse Hydroponic Farms using IOT", International Journal for Research in Applied Science \& Engineering Technology ,2018, Volume 6 ,Issue 3.

[4]Nisha sharma, Somen acharya, Kaushal kumar, Narendra singh,O.P. chaurasia, "Hydroponics as an advanced technique for vegetable production: An overview “, Journal of Soil and Water Conservation ,2018pp: 364-371.

[5] Mitali V. Shewale , Devendra S.Chaudhari," IoT based Plant Monitoring System for Hydroponics Agriculture: a Review," International Journal for Research in Applied Science \& Engineering Technology,2018, Volume 6, Issue 2.

[6] Chanya Peuchpanngarm, Srinitiworawong, Wannisa Samerjai,Thanwadee Sunetnanta, "DIY SensorBased Automatic Control Mobile Application for Hydroponics", IEEE 5th ICT International Student Project Conference, 2016 .

[7] S. Umamaheswari, A. Preethi, E. Pravin, R. Dhanusha, "Integrating Scheduled Hydroponic System", IEEE International Conference on Advances in Computer Applications, 2016.

[8] Jumras Pitakphongmetha, Nathaphon Boonnam, Siriwan Wongkoon, Teerayut Horanont, "Internet of Things for Planting in Smart Farm Hydroponics Style", IEEE International Conference on Advances in Computer Applications, 2016.

[9] Mahesh, Minhas Naheem, RazakMubafar, Shyba, Sunitha Beevi, "New Aspect for Organic Farming Practices: Controlled Crop Nutrition and Soilless Agriculture", IEEE Global Humanitarian Conference 2016 .

[10] Chavan Akshay , Pawar Abhijeet , Wagh Pratik , Lalita Wani,“ IOT Based Hydroponic System”, International Journal of Innovative Research in Computer and Communication Engineering, 2017,Vol. 5, Issue 4.

[11] S. Suakanto, V. J. L. Engel, M. Hutagalung, D. Angela, "Sensor networks data acquisition and task management for decision support of smart farming," International Conference on Information Technology Systems and Innovation, 2016, pp. 1-5. 
[12] A. Kamilaris, F. Gao, F. X. Prenafeta Boldu, M. I. Ali, "Agriiot- A semantic framework for internet of things enabled smart farming applications," IEEE 3rd World Forum on Internet of Things , 2016, pp. $442-447$.

[13] A. Kaloxylos, R. Eigenmann, F. Teye, Z. Politopoulou, S. Wolfert, C. Shrank, M. Dillinger, I. Lampropoulou, E. Antoniou, L. Pesonen, H. Nicole, F. Thomas, N. Alonistioti, G. Kormentzas, "Farm management systems and the future internet era," Computers and Electronics in Agriculture, 2012,vol. 89, pp. $130-144$.

[14] ]V. S. Kumar, I. Gogul, M. D. Raj, S. Pragadesh, J. S. Sebastin, "Smart autonomous gardening rover with plant recognition using neural networks," Procedia Computer Science, 2016,vol. 93, pp. 975 - 981.

[15] Melchizedek I. Alipio, Allen Earl M. Dela Cruz, Jess David A. Doria,Rowena Maria S. Fruto, “A Smart Hydroponics Farming System Using Exact Inference in Bayesian Network ",IEEE 6th Global Conference on Consumer Electronics,2017.

[16] Jumras Pitakphongmetha, Nathaphon Boonnam, Siriwan Wongkoon, Teerayut Horanont Deeprom Somkiadcharoen, Jiranuwat Prapakornpilai, "Internet of Things for Planting in Smart Farm Hydroponics Style", 2016.

[17]Mr. Patil Anil, Sabale Snehal, "Hydroponics farming using IOT", International journal of basic and applied research, 2019, Volume 9 Number 5. 